\section{Talking telephone numbers}

SIR - Your comments on telephone numbers (Nature 358, 440; 1992) oversimplify the situation.

(1) The currently normal 10-digit string we dial is actually a 9-digit identifier preceded by a single-digit access code. There is a trade-off in the time taken to use an access code: if it were not used to select out-of-area calls, it would be necessary to dial 9 digits even for the local calls for which we normally use 6 or 7 digits.

(2) Historically the leading digits had geographical significance, but their importance now is that they carry charging information. Until the day arrives that all standard point-to-point calls within the United Kingdom are charged at the same rate, it will be necessary to allocate number ranges to geographical areas. In addition, other number ranges $(800,345$, 500,898 and others) attract specific charge rates independent of destination. The cost to business of not being able to monitor telephone bills by checking the codes dialled could well outweigh the cost of using an extra digit.

(3) In the longer run, the extra digit can be regarded as a selector of both carrier (British Telecom, Mercury or other) and service (for example mobile telephones, personal location-independent numbers) and so it may become the primary indicator of charge rate.

(4) Already in parts of the United States there are more telephone numbers than people so the $10^{8}$ limit you suggest for the United Kingdom is likely to be exceeded early in the next century.

To summarize, the main factor in the inefficient use of the numbering range is not the need for geographical information as such, nor the historical limitations imposed by old technology, but the unwillingness of the consumer (and Oftel, the regulatory body) to accept that with current technology almost all the cost of a call arises in the local loop, and so is independent of the distance to the called party.

\section{John Littler}

Telecom Potential,

Clevedon Hall, Victoria Road,

Clevedon, Avon BS21 7RQ, UK

SIR - As you point out, modern electronic switching works equally well with any sequence of digits, and telephone companies would have more numbers available to them if they didn't have to reserve specific sequences for specific areas.

What you did not consider, however, was who is on the other end of all that modern electronic switching. Even in this era of faxes and modems, most telephone calls are still placed by hu- mans, who have to try to remember an ever increasing number of telephone numbers. Humans, unlike computers, remember numbers better when the numbers are in groups and when the groups have some sort of reference. Thus, 912027372355 (the telephone number of Nature's Washington office) is almost impossible to remember unless we take it in groups: 9 (that gets me an outside line), 1 (long distance), 202 (the Washington DC area code), 737 (the exchange), 2355 (the number). To remember this number, all I have really to remember is Washington, the exchange, and the number. Area codes and exchanges I call frequently are easy to associate with specific geographical areas and thus easier to remember than random sequences. The scheme you suggest would make it much harder on us poor humans.

\section{Michael Blechner}

AAPG Publications,

PO Box 979

Tulsa, Oklahoma 74101-0979, USA

\section{Consciousness}

SIR - The discussion of consciousness ${ }^{1-3}$ suggests the lack of a consistent definition. Consciousness is clearly a result of neuronal activity, because it is suspended by sleep, mechanical trauma and drugs such as anaesthetics. As we humans evolved from other mammals, our brains are basically the same as theirs, so that the question also arises of whether consciousness differs qualitatively or merely quantitatively from the brain activity of apes or dogs. Unfortunately, they cannot tell us whether they are conscious. But this highlights my conception of consciousness, the ability to talk silently to myself.

Language is unique to humans, and all our civilization depends on it. Teachers know that full understanding of a subject often comes only when it has to be put into words in order to communicate that understanding to students. We are capable of transmitting and receiving complex information encoded in abstract acoustic or visual signals (talking and reading). Suppose that an individual's brain encodes the information it is 'aware of' into language, and then interprets the stream of chosen words much as if it came from an external source, checking the accuracy of the description, and possibly generating corrections. That activity would seem to fulfil the basics of consciousness.

Let me suggest a model: individual A keeps up a running commentary on his actions and perceptions to individual $\mathrm{B}$, who listens, interprets and, when he feels like it, replies with observations and suggestions that $\mathrm{A}$ uses to modify his actions and perceptions. B is effectively the consciousness of $\mathrm{A}$. If $\mathrm{A}$ and $\mathrm{B}$ are the same person, we have a conscious person. Within one brain, the communication could include neuronal representations of phenomena as well as abstract language, which would be available to other animals. But only humans have the subtlety of language to finetune the process. This does not solve the problem of consciousness but makes human consciousness a consequence of the development of language, as Jaynes ${ }^{4}$ has argued.

Hugh L. Fletcher

Queens University,

Division of Genetic Engineering,

97 Liston Road, Belfast BT9 $7 B L$,

Northern Ireland, UK

1. Gray, J. Nature 358, 277 (1992)

2. Josephson, B. D. et al. Nature 358, 618 (1992)

3. Sacks, O. Nature 358, 618 (1992).

4. Jaynes, J. The Origin of Consciousness and the Breakdown of the Bicameral Mind (Houghton Mifflin Boston, 1990).

\section{True or false?}

SIR - Writing about my book The Facts of Life: Shattering the Myth of Darwinism, the author of your leading article (Nature 358, 698; 1992) says that I believe evolution to be false, that natural selection is a 'pack of lies' and that I believe the Earth to be young. Any objective reader of my book will find that none of these statements is true. I accept evolution; but I present evidence that the neo-Darwinian mechanism cannot wholly account for it. I do not think natural selection a pack of lies; I present evidence that it is incapable of leading to trans-specific evolution. And I do not claim the Earth is young, but present evidence that currently accepted methods of geochronometry are seriously flawed and should be re-examined.

Instead of addressing the scientific issues raised by this evidence, you dismiss it simply by placing quotation marks around the word evidence, implying that what I have written is not really evidence.

I am a professional science reporter and if I have misrepresented any of the scientific work referred to I shall be glad to receive specific details.

Richard Milton

Beech Hurst,

10 Pembury Road,

Tonbridge, Kent, UK

$\square$ So why does the book jacket proclaim that the book contains " $\mathrm{t}]$ he evidence against the Darwinian idea that chance is the mechanism of evolution"? Editor, Nature. 